\title{
Le contrôle de la recherche d'emploi et les sanctions
}

Job search monitoring and sanctions

\section{Gwenn Parent}

\section{OpenEdition}

\section{Journals}

Édition électronique

URL : http://journals.openedition.org/travailemploi/6438

DOI : 10.4000/travailemploi.6438

ISSN : 1775-416X

Éditeur

DARES - Ministère du Travail

\section{Édition imprimée}

Date de publication : 1 juillet 2014

Pagination : $91-104$

ISSN : 0224-4365

\section{Référence électronique}

Gwenn Parent, «Le contrôle de la recherche d'emploi et les sanctions », Travail et Emploi [En ligne], 139 | juillet-septembre 2014, mis en ligne le 01 juillet 2016, consulté le 19 juin 2020. URL : http:// journals.openedition.org/travailemploi/6438; DOI : https://doi.org/10.4000/travailemploi.6438 


\title{
Le contrôle de la recherche d'emploi et les sanctions
}

\author{
Gwenn Parent ${ }^{(*)}(* *)$
}

Un contrôle de la recherche d'emploi permet de vérifier qu'un demandeur d'emploi s'est engagé dans des activités de recherche d'emploi suffisantes pour pouvoir continuer à percevoir des allocationschômage. Une sanction est une réduction voire une suppression de l'indemnisation, lorsqu'il est avéré qu'un chômeur a commis une infraction et n'a pas respecté les exigences formulées par le service public de l'emploi (SPE). Cet article présente une revue de littérature des principales expériences européennes et évaluations microéconométriques de ces contrôles et des sanctions correspondantes. Les résultats de ces évaluations montrent que les contrôles comme les sanctions ont des effets positifs sur le retour à l'emploi, mais négatifs sur la qualité des emplois retrouvés. Ces mécanismes d'activation inciteraient donc les demandeurs d'emploi à accepter des emplois moins stables et moins bien rémunérés. Ils peuvent également avoir des effets pénalisants sur le parcours professionnel ultérieur des personnes sanctionnées, et conduire à des effets d'éviction vers diverses formes d'inactivité.

L'obligation pour les chômeurs de rechercher un emploi et le contrôle de l'effectivité de ces recherches ont accompagné la création des systèmes d'indemnisation du chômage dans la plupart des pays. C'est le cas en France, depuis les premières formes d'indemnisation du chômage avec la création des caisses syndicales à la fin du XIX ${ }^{e}$ siècle, puis du Fonds national de chômage en 1914. Le décret du 12 mars 1951 sur l'aide aux travailleurs sans emploi prévoit déjà les cinq motifs d'exclusion suivants : "absence de réponse aux convocations, refus d'un emploi offert par les services de maind'œuvre, refus de fréquenter les cours de formation professionnelle conseillés par les services de maind'œuvre, fraude aux allocations et le fait de se livrer habituellement à la boisson. » L'aide publique allouée a ainsi toujours été étroitement conditionnée à la recherche d'un emploi et à des contrôles autoritaires : la «suspicion à la fraude des chômeurs» a accompagné de fait, tout au long du $\mathrm{Xx}^{\mathrm{e}}$ siècle, le débat entre assistance et assurance des chômeurs (Magniadas, 2008).

Les contrôles et les sanctions se sont développés dans les années 1970 aux États-Unis et dans les années 1980 en Europe, où ils se sont généralisés au début des années 2000. Avec la crise économique

(*) Direction de l'animation de la recherche, des études et des statistiques (Dares) au moment de la rédaction de cet article; gwenn.parent@gmail.com

(**) Cet article s'appuie sur le document d'études de PARENT et al. (2013) publié par la Dares. Je remercie Céline Gratadour, Olivia Sautory, Oana Calavrezo et Rozenn Desplatz pour leur participation à ce projet ainsi que Cyril Nouveau et Christel Colin pour leur relecture attentive de ce document d'études et leurs conseils avisés. des années 1970, les offres d'emploi sur le marché du travail se sont raréfiées, le taux de chômage a progressé rapidement dans l'ensemble des pays européens, augmentant ainsi de manière concomitante le nombre de bénéficiaires d'allocations-chômage. Dans ce contexte dégradé, la théorie économique s'est intéressée plus particulièrement à l'analyse des effets potentiellement désincitatifs de l'indemnisation du chômage et aux moyens de les limiter. Milton Friedman (1962) comme les économistes ayant développé différents modèles de prospection d'emploi (modèles de job search; Stigler, 1962), mettent en exergue certains effets désincitatifs du système d'allocation-chômage, qui découragerait le retour à l'emploi des chômeurs en diminuant les gains à la reprise d'activité. Sur le plan théorique, l'indemnisation financière des demandeurs d'emploi engendre en effet d'importants problèmes d'aléa moral : les allocations-chômage augmentent le salaire à partir duquel les personnes indemnisées acceptent un emploi (salaire de réserve ou de réservation) et peuvent également les inciter à réduire leurs efforts de recherche d'emploi. Les allocations-chômage peuvent ainsi, en théorie, avoir un impact négatif sur le retour à l'emploi. Cependant, les éventuels effets désincitatifs peuvent être limités par une stratégie d'activation des demandeurs d'emploi consistant soit à contrôler l'effectivité de leurs recherches d'emploi, soit à imposer des sanctions (réduction ou suspension des allocations-chômage) en cas d'efforts insuffisants, soit encore à offrir une prime en cas de reprise d'emploi. Les mécanismes théoriques présentés dans la suite de l'article s'inscrivent dans ce cadre conceptuel. 
D'autres approches posent à l'inverse que les allocations-chômage peuvent améliorer la recherche d'emploi en finançant des actes de recherches coûteux, et surtout peuvent améliorer la qualité des appariements en permettant aux chômeurs d'attendre des offres de meilleure qualité (ACEMOGLU, 2001). Dans ce cadre, le filet de sécurité ainsi proposé aux demandeurs d'emploi, qui est davantage développé dans les pays européens que dans d'autres pays de l'Organisation de coopération et de développement économiques (OCDE, 2006, 2009, 2012), permet de lisser les revenus des personnes momentanément sans emploi et de limiter les transitions vers la pauvreté lors de ces accidents de parcours professionnel.

Récemment, la mise en œuvre de stratégies d'activation des demandeurs d'emploi, basées sur le principe d'obligations mutuelles ${ }^{(1)}$ a relancé le débat sur la nécessité de contrôler plus activement les chômeurs et d'appliquer des sanctions lorsque ces obligations ne sont pas respectées. Cependant, si la mise en œuvre de politiques de contrôles et de sanctions est devenue la norme en Europe, leur évaluation est restée très inégale selon les pays. Ainsi, aucune évaluation des contrôles ou des sanctions n'a été réalisée en France. En revanche, d'autres pays comme la Belgique, les Pays-Bas, la Suisse, l'Allemagne ou le Danemark ont mis en place des protocoles d'évaluation expérimentaux permettant une réelle analyse d'impact. Ces pays disposent souvent de données administratives ou d'enquêtes suffisamment riches pour permettre une analyse précise des mécanismes à l'œuvre lors de l'imposition de contrôles ou de sanctions. Certes, la fréquence et l'objet des contrôles, comme les conditions d'application des sanctions, sont très différents d'un pays à l'autre, ce qui limite les préconisations et les enseignements qui peuvent en être tirés dans d'autres cadres institutionnels.

Cet article s'organise en deux parties : la première présente les évaluations d'impact des contrôles de la recherche d'emploi alors que la seconde analyse les évaluations portant sur les sanctions. Les deux parties décrivent successivement les effets observés sur les taux d'emploi et sur la qualité des emplois retrouvés, les principaux enseignements relatifs à la mise en œuvre de ces politiques et, enfin, discutent l'existence d'effets de substitution ou d'effets d'éviction. La conclusion synthétise les principales conclusions de la revue de littérature proposée.

(1) La généralisation des plans d'actions individuels a permis de formaliser précisément et de contractualiser ces obligations mutuelles entre le demandeur d'emploi et le SPE, pour servir de socle de référence pour les contrôles et sanctions éventuelles ultérieures.

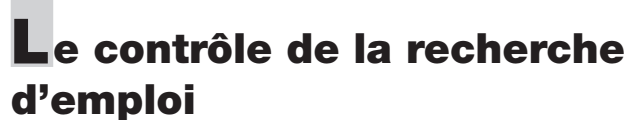

Les contrôles de la recherche d'emploi visent à vérifier que les demandeurs d'emploi se sont effectivement engagés dans des activités de recherche d'emploi conformes aux exigences du SPE et donc suffisantes pour pouvoir continuer à percevoir une allocation-chômage. Les contrôles peuvent également porter sur la vérification de la disponibilité du demandeur d'emploi ou sur les éventuels refus d'«offres raisonnables d'emploi» (ORE). Les critères d'acceptabilité d'une offre d'emploi varient d'un pays à l'autre, tant en termes de mobilité géographique que de salaire ou de qualification ${ }^{(2)}$.

\section{Les effets théoriques attendus des contrôles}

Les modèles théoriques de prospection d'emploi (parmi lesquels Mortensen, 1986; van DEN BerG, 1990) prédisent que les bénéficiaires d'allocationschômage modifient l'intensité de leurs recherches d'emploi et leur comportement d'acceptation des offres lorsqu'un événement affecte leur niveau d'indemnisation (par exemple, contrôle des recherches d'emploi effectuées ou situation de fin de droits à l'indemnisation du chômage). Le contrôle de la recherche d'emploi peut ainsi avoir des effets sur le retour à l'emploi des demandeurs d'emploi avant même d'intervenir réellement : on parle alors d'effet de menace du contrôle. La plupart des articles théoriques présentent des modèles dans lesquels l'effort de recherche d'emploi augmente avec les exigences de recherche requises pour bénéficier de l'assurance-chômage, ainsi qu'avec la probabilité d'être contrôlé prochainement. En théorie, l'augmentation des efforts de recherche se traduit par une multiplication du nombre des contacts avec les employeurs et, ce faisant, par une augmentation de la probabilité de retrouver un emploi. Les contrôles augmentent donc théoriquement les taux de reprise d'emploi et réduisent la durée des épisodes de chômage.

Gerard Van den Berg et Bas van der KlaAuw (2006) identifient un second effet théorique des contrôles. Les auteurs évaluent l'efficacité comparée en termes de retour à l'emploi de différents canaux de recherche d'emploi : les canaux formels de recherche d'emploi, qui peuvent être contrôlés (visites à l'agence, nombre de candidatures envoyées en réponse à des offres formalisées, etc.), et les canaux informels (comme la sollicitation de réseaux

(2) Pour plus de détails sur les critères d'éligibilité à l'assurance-chômage, les critères requis pour qu'une offre soit considérée comme acceptable, la fréquence des contrôles de recherche d'emploi et les modalités de sanctions, voir Danielle VENN (2012), qui présente un tour d'horizon détaillé de ces aspects dans trente-six pays membres de l'OCDE et/ou de l'Union européenne (dont la France). 
personnels ou de réseaux sociaux) qui, par nature, ne sont pas observables et ne peuvent donc pas être contrôlés par les conseillers. Le conseil offert dans le cadre des politiques actives du marché du travail (PAMT, auxquelles est consacré ce numéro de la revue Travail et emploi) vise avant tout à intensifier le premier mode (formel) de recherche d'emploi, qui est plus facilement observable et contrôlable. Les auteurs montrent que l'introduction d'un système de contrôle des recherches d'emploi peut théoriquement conduire à une substitution des canaux de recherche, de l'informel vers le formel, puisque les efforts de recherche via des canaux informels ne peuvent être vérifiés par le conseiller, et peuvent donc conduire ce dernier à une évaluation négative des efforts réalisés ( $c f$. infra pour les évaluations empiriques). Ces canaux observables n'étant pas forcément les plus efficaces, le contrôle peut de ce fait, au moins en théorie, réduire l'efficacité des recherches effectuées.

\section{Résultats des évaluations empiriques des contrôles}

Les États-Unis ont été précurseurs dans l'évaluation du contrôle de la recherche d'emploi. En effet, depuis sa mise en place en 1930, le système américain d'indemnisation du chômage a suscité de nombreuses controverses en raison de ses effets désincitatifs supposés. Des projets de recherche, expérimentaux et non expérimentaux, ont été financés par le ministère du Travail américain au cours des années 1980 pour évaluer les effets des contrôles sur la réduction de ces désincitations ${ }^{(3)}$. À partir des années 1990, les dispositifs de contrôle se développent au sein des pays de l'OCDE (OCDE, 2000). Les États-Unis et certains pays d'Europe du Nord mettent en place dans ce cadre des programmes expérimentaux dont les bénéficiaires sont sélectionnés aléatoirement ${ }^{(4)}$, ce qui permet d'évaluer plus finement leurs effets ${ }^{(5)}$. Bien que non systématiques ${ }^{(6)}$, d'autres évaluations sont réalisées en

(3) Exploitant les différences de politiques mises en œuvre dans les différents États américains en matière de contrôle de la recherche d'emploi, les résultats de ces premières études vont dans le sens d'un effet positif d'exigences plus sévères quant aux recherches d'emploi et à leurs contrôles sur le retour à l'emploi des chômeurs (voir AsHENFELTER et al. [2005] pour une présentation détaillée de ces premières études, qui souffrent néanmoins de sérieux problèmes méthodologiques [MEYER, 1995]).

(4) Pour en savoir plus sur le sujet, voir le numéro 135 de Travail et emploi : LABRousse, Zamora (coord.), 2013.

(5) Dans la suite de l'article, nous présentons les résultats de certains de ces programmes précurseurs au niveau européen comme par exemple " Jobseeker's Allowance» mis en place au Royaume-Uni en 1996 ou «Counseling and Monitoring» lancé aux Pays-Bas en 1998.

(6) Ainsi, aucune évaluation spécifique des contrôles n'a été réalisée en France, quand d'autres pays comme la Belgique, avec le programme «Activation du comportement de recherche» (ACR) mis en place en 2004, ou le Danemark, avec une expérimentation sociale menée en 2005-2006, ont adopté des protocoles d'évaluation expérimentaux permettant une réelle analyse d'impact. Les résultats de ces évaluations sont également présentés.
Europe dans les années 2000, quand les contrôles de la recherche d'emploi se généralisent.

Cependant, peu d'articles empiriques traitent exclusivement de l'évaluation du contrôle. Jusqu'à récemment, la plupart des programmes mis en œuvre associent en effet des contrôles renforcés avec, soit des services complémentaires d'aide à la recherche d'emploi (accompagnement), soit des sanctions en cas de non-conformité des efforts fournis par rapport aux exigences du SPE. Il est ainsi difficile d'identifier de façon précise laquelle de ces composantes est à l'origine des plus ou moins bons résultats observés sur le retour à l'emploi.

\section{Les effets sur le retour à l'emploi et la qualité des emplois retrouvés}

Si quelques études, comme par exemple les travaux réalisés par AsHenfelter et al. (2005)(7), mettent en avant des résultats mitigés, la grande majorité conclut à des résultats positifs sur le retour à l'emploi.

Ainsi Rosholm et al. (2011) dans leur évaluation de l'expérimentation sociale menée en 2005-2006 au Danemark notent que l'association entre des entretiens fréquents avec un conseiller et des contrôles intensifs des recherches réalisées ont un effet positif sur la probabilité de transition vers l'emploi, bien qu'il ne soit pas toujours possible d'isoler l'effet propre du contrôle de celui des conseils apportés en entretien.

S'appuyant sur les données collectées lors des périodes de rénovation des locaux ${ }^{(8)}$ de certaines agences pour l'emploi en Irlande du Nord entre 1999 et 2005 - au moment de la mise en place de guichets uniques (regroupant les fonctions d'indemnisation et d'accompagnement) -, Duncan Mc VICAR (2008) recourt à des méthodes quasi expérimentales pour mesurer l'impact des contrôles de la recherche d'emploi. Pendant ces périodes de réorganisation des agences locales ${ }^{(9)}$, les contrôles des recherches effectuées par les bénéficiaires d'al-

(7) Sur la base d'expérimentations réalisées entre 1984 et 1985 dans quatre États américains, AsHENFELTER et al. (2005) soulignent que la procédure consistant à vérifier les démarches que les chômeurs déclarent avoir faites avant même de commencer à leur verser des prestations a permis de réduire de $8 \%$ le taux d'ouverture des droits à indemnisation ("Qualification Rate») dans le Connecticut, mais qu'elle n'a eu aucun effet dans les trois autres États (Massachusetts, Tennessee, Virginie). De tels résultats conduisent les auteurs à porter un jugement très nuancé sur l'efficacité d'un contrôle accru de la recherche d'emploi, dispositif aux résultats limités au regard du coût important engendré.

(8) De fait, ces rénovations ont eu pour conséquence de devoir supprimer les contrôles par manque de locaux disponibles.

(9) Ces périodes sont considérées comme exogènes car elles interviennent de manière non corrélée ni aux politiques de contrôle des chômeurs ni à leur timing. Cette exogénéité permet aux auteurs de construire des expériences naturelles (voir l'article de Gwenn Parent et Olivia Sautory dans ce même numéro). 
location-chômage ${ }^{(10)}$ sont suspendus, mais pas les exigences de recherche, ni les aides à la recherche d'emploi : le contrôle seul étant suspendu (pendant huit mois en moyenne dans chaque agence), l'auteur peut considérer la mise en place des guichets uniques comme une expérience naturelle. Grâce à une méthode quasi expérimentale, il montre alors que la suspension du contrôle augmente la durée moyenne des épisodes de chômage de $16 \%$ et réduit la probabilité de transition vers l'emploi de $26 \%$.

À partir de 2004, la Belgique met en place le dispositif "Activation du comportement de recherche» (ACR), qui consiste en des entretiens individuels périodiques avec un conseiller, dont le but est d'évaluer les activités de recherche d'emploi des chômeurs. En cas de non-respect des exigences posées, des sanctions sont appliquées (réduction ou suspension temporaire ou définitive de l'allocation). L'ACR et le contrôle de la disponibilité des chômeurs sont considérés comme des contreparties nécessaires à la spécificité belge d'indemnisation du chômage pour une durée illimitée (COCKX et al., 2011b). Les personnes en recherche d'emploi depuis plus de treize mois sont informées que leurs recherches seront contrôlées huit mois plus tard (11), ce qui permet d'identifier les effets de menace du contrôle. Bart Cockx et Muriel Dejemeppe (2007, 2010), ainsi que Cockx et al. (2011a, 2011b), exploitent la discontinuité de l'application de ce programme autour de l'âge de 30 ans ${ }^{(12)}$. L'ACR augmente sensiblement les taux de reprise d'emploi des chômeurs avant même que les entretiens individuels avec les conseillers n'aient lieu et qu'une sanction soit éventuellement appliquée (CockX, Dejemeppe, 2007). Cockx et Dejemeppe (2010) trouvent que l'effet pur de menace de contrôle augmente les transitions vers l'emploi : en Flandre, huit mois après la réception de la lettre d'avertissement et avant la vérification effective des efforts de recherche, le taux de transition vers l'emploi est de l'ordre de dix points de pourcentage (six points en Wallonie) plus élevé qu'en l'absence de l'ACR, ce qui correspond à une augmentation de $28 \%(22 \%$ en Wallonie) du taux de transition vers l'emploi selon les auteurs. L'impact plus important en Flandre

(10) Ces contrôles étaient au préalable réalisés lors d'entretiens spécifiques par des conseillers différents de ceux qui étaient chargés de l'aide à la recherche d'emploi.

(11) L'échantillon comprend les personnes ayant atteint leur treizième mois de chômage en 2004, qui reçoivent alors une lettre de notification des contrôles à venir. La période d'observation s'étend ensuite de 2004 à 2006. Compte tenu de la durée prévue entre les différentes étapes de l'ACR (au moins huit mois entre la lettre et le premier entretien, et quatre mois entre les entretiens ultérieurs), beaucoup de chômeurs ont déjà quitté le chômage indemnisé avant de participer aux entretiens. Aussi, un peu plus de la moitié des chômeurs qui ont été avertis au cours d'une période donnée participent effectivement à un premier entretien d'évaluation.

(12) Seules les personnes de 25 à 29 ans font partie du programme dans un premier temps, la généralisation aux 30-34 ans n'intervenant que dans un second temps. est probablement lié aux conditions économiques alors plus favorables qu'en Wallonie. De plus, si la Wallonie a mis en place parallèlement une aide importante à la recherche d'emploi, l'effet trouvé dans le cas de la Flandre est considéré comme un effet pur du contrôle car aucune aide supplémentaire n'était apportée aux demandeurs d'emploi. Cockx et al. (2011a) approfondissent l'étude en évaluant toutes les étapes du dispositif ACR et non seulement l'effet de menace. Ils notent que cette réforme du contrôle de la recherche d'emploi incite les chômeurs à concentrer leurs recherches juste avant le moment du contrôle, avec pour conséquence d'augmenter le taux de retour en emploi et de l'amener à un niveau même supérieur à celui qu'il atteint après l'application d'une sanction. Le contrôle de la recherche d'emploi renforce l'effort de recherche ex ante, mais le réduit ex post ${ }^{(13)}$. Cette intensification des recherches d'emploi par les demandeurs d'emploi réduit donc la durée des épisodes de chômage. Afin de réduire l'aléa moral créé par la durée illimitée de l'indemnisation belge du chômage, le renforcement des contrôles de la recherche d'emploi semble ainsi constituer une alternative crédible et efficace à la limitation de la durée de l'indemnisation, proposition qui est alors au cœur du débat public en Belgique depuis plusieurs années.

Ces effets globalement positifs des contrôles sur le retour à l'emploi doivent être nuancés par ceux, plutôt négatifs, que la littérature met en évidence sur la qualité des emplois. Il semble qu'ils peuvent néanmoins être contrés par un accompagnement dans la recherche d'emploi (14). L'effet sur les salaires des emplois retrouvés est plus ambigu.

Cockx et Dejemeppe (2007), dans leur analyse du dispositif belge ACR, évaluent l'impact de la réforme sur la qualité des emplois retrouvés. En Flandre, les personnes en emploi informées des contrôles et éventuelles sanctions à venir sont dans des emplois moins stables (autrement dit, la proportion de personnes toujours en emploi trois ou six mois après être sortis du chômage est plus faible) et plus souvent à temps partiel que les personnes en emploi du groupe de contrôle. Les auteurs mettent ainsi en garde contre le risque d'acceptation d'emplois de moins bonne qualité. Cependant, le dispositif n'était pas accompagné en Flandre d'aide particulière à la recherche d'emploi, contrairement à ce qui était pratiqué en Wallonie et à Bruxelles. Dans ces régions, aucun effet négatif sur la qualité des emplois n'est identifié, ce qui permet aux auteurs de suggérer que le risque de dégradation de la qualité

(13) Les auteurs n'expliquent pas cette réduction de l'effort de recherche ex post, qu'ils voient comme un sujet de recherches futures.

(14) Voir l'article de G. Parent et O. Sautory de ce même numéro pour une revue de littérature des effets de l'accompagnement des demandeurs d'emploi. 
des emplois retrouvés peut être contré par un timing approprié pour le conseil à la recherche d'emploi. CockX et al. (2011a) montrent par ailleurs que les salaires des emplois retrouvés ne sont affectés que marginalement par la mise en place du dispositif ACR (moins de $2 \%$ de baisse en moyenne par rapport aux personnes non soumises aux contrôles).

VAN DER BERG et VAN DER KLAAUw (2013) évaluent le programme "Counseling and Monitoring" (C\&M) mis en place aux Pays-Bas en 1998 pour des bénéficiaires ayant de relativement bonnes chances de retour à l'emploi ${ }^{(15)}$. Dans le cadre de ce programme, des conseils en matière de recherche d'emploi et une surveillance accrue de la recherche sont prodigués aux chômeurs considérés comme les plus employables, ce pendant six mois avec un rendez-vous en agence toutes les quatre semaines. Il ressort de l'évaluation que le programme C\&M a certes légèrement réduit la durée des épisodes de chômage, mais qu'il a dans le même temps incité les demandeurs d'emploi à accepter des emplois de moins bonne qualité (emplois de plus courte durée et aux salaires inférieurs). Barbara Petrongolo (2009) obtient des résultats similaires concernant la mise en place de la "Jobseeker's Allowance» (JSA) au Royaume-Uni en 1996 : elle démontre que le renforcement des exigences attendues en matière de recherches d'emploi et leur contrôle accru ont substantiellement réduit la stabilité dans l'emploi retrouvé ainsi que les revenus annuels à venir, et ce sur le long terme ${ }^{(16)}$.

\section{La mise en guvre et le timing des contrôles}

Les questions de mise en œuvre des politiques de contrôles de la recherche d'emploi jouent un rôle crucial dans la nature des effets constatés sur le retour à l'emploi ou la qualité des emplois retrouvés.

VAN DEN Berg et VAn DER KlaAuw (2006, 2013) montrent que les canaux de recherche d'emploi (formel / informel) sont modifiés par les contrôles du programme C\&M aux Pays-Bas. L'analyse des données collectées prouve que les chômeurs ont fait évoluer leur effort de recherche d'emploi et se sont tournés vers des modes formels de recherche, au détriment de modes plus informels. Pour les populations pour lesquelles le canal informel s'avère plus efficace pour le retour à l'emploi (les personnes les plus qualifiées), les auteurs démontrent que 1'introduction d'un système de contrôle peut ainsi se révéler inefficace, et même dans certains cas contreproductif. Les résultats de Mc VICAR (2008), qui s'appuient sur les périodes de réorganisation des agences de 1'emploi en Irlande du Nord (cf. supra),

(15) L'évaluation repose sur une expérimentation contrôlée de taille modeste avec orientation aléatoire vers le programme (l'échantillon est composé de 393 chômeurs indemnisés).

(16) Les écarts de salaire sont significatifs même plusieurs années après la mise en place de la JSA. suggèrent néanmoins que l'effet positif du contrôle de la recherche via des canaux formels est plus important que l'effet négatif de substitution de la recherche informelle. Les différences de résultats entre ces deux études peuvent s'expliquer par les profils différents des bénéficiaires étudiés (17) : l'analyse empirique de VAN DEN BERG et VAN DER KLAAUw porte en effet sur les chômeurs de type I aux PaysBas ${ }^{(18)}$, les plus proches du marché du travail, tandis que l'étude de Mc VICAR concerne l'ensemble des demandeurs d'emploi inscrits et indemnisés. Le mécanisme de substitution des canaux de recherche d'emploi qui réduit l'effet des contrôles concernerait donc surtout les chômeurs les plus susceptibles de retrouver seuls un emploi.

L'une des questions essentielles qui se posent aux SPE dans la mise en œuvre d'une politique de contrôle est de savoir quel élément contrôler : l'intensité des recherches d'emploi, la disponibilité du chômeur pour occuper un emploi, suivre une formation ou un programme d'accompagnement ou bien le refus des «offres raisonnables d'emploi» (ORE). VAN DER BERG et VIKSTRÖM (2014) développent une modélisation théorique de prospection d'emploi (modèle de Job Search) et montrent que le contrôle des refus d'offres d'emploi est moins efficace que celui de l'effort de recherche d'emploi. Cockx et al. (2011a) concluent, de leur évaluation de l'ACR en Belgique, que ces deux types de contrôles sont complémentaires car leurs objets (respectivement le caractère «convenable» d'une offre d'emploi et l'intensité des recherches d'emploi) sont tous deux imparfaitement observés.

Les SPE s'interrogent également sur le timing le plus approprié pour les contrôles. Lorsque l'on tient compte de leur coût, qui peut être potentiellement élevé si ceux-ci sont appliqués dès le début de l'épisode de chômage (et concernent donc un nombre important de chômeurs), il semble qu'un contrôle trop précoce ne soit pas efficient. Conny Wunsch (2010) ${ }^{(19)}$ montre que l'intensité du contrôle doit être relativement faible au début de l'épisode de chômage et s'intensifier au fur et à mesure que

(17) Une autre explication est avancée dans le cas du programme C\&M aux Pays-Bas : la faible intensité de l'aide à la recherche d'emploi fournie aux demandeurs d'emploi ( $c f$. article de G. Parent et O. Sautory de ce même numéro).

(18) Aux Pays-Bas, entre 75 et $80 \%$ du flux de nouveaux bénéficiaires de l'indemnisation chômage sont classés en type I (chômeurs ayant la capacité de retrouver un emploi sans assistance particulière). Les trois autres types (de II à IV) sont supposés nécessiter davantage d'aide et d'accompagnement. Le mécanisme de profilage permettant de classer les chômeurs dans l'un de ces quatre types repose sur une mesure de leur distance au marché du travail (probabilité estimée de reprise d'emploi), distance censée donner une indication quant à la durée probable de chômage (PARENT et al. [2013] comporte un chapitre entier dédié à ces méthodes).

(19) Cet article s'appuie sur un modèle théorique, qui est calibré sur des données de l'Allemagne de l'Ouest et prend en compte le coût du contrôle. 
la durée de chômage s'allonge. De façon optimale, l'intensité du contrôle doit augmenter à mesure que diminue le rendement des recherches effectuées (nombre d'entretiens d'embauche obtenus par candidature) afin de réinstaurer une incitation à la recherche : en effet, l'augmentation de la durée passée au chômage entraîne dans ce modèle une diminution des salaires à l'embauche et des taux de reprise d'emploi. Empiriquement, comme nous l'avons indiqué plus haut, COCKX et DeJEMEPPE (2010) obtiennent des évaluations divergentes de l'ACR selon la région considérée : si le programme exerce un effet positif sur le retour en emploi en Wallonie et en Flandre, la notification d'un prochain contrôle qu'il prévoit a un effet opposé à Bruxelles. D'après les auteurs, ces écarts entre régions reflètent les différentes manières dont les SPE régionaux ont mis en place la réforme. L'effet négatif observé à Bruxelles pourrait notamment être dû au timing inapproprié de l'aide à la recherche d'emploi accompagnant le renforcement du contrôle dans cette région : elle lui est postérieure et a pu en inverser l'effet de menace, les chômeurs ayant préféré attendre le contrôle afin d'être conseillés dans leurs démarches de recherche d'emploi (effet d'attraction de l'accompagnement $\left.{ }^{(20)}\right)$. Les auteurs concluent que le timing de l'aide à la recherche d'emploi fournie aux chômeurs est capital et comporte des effets incitatifs importants qui peuvent modifier les effets des contrôles.

Il apparaît donc nécessaire de mettre en œuvre les contrôles de manière subtile, en les adaptant aux publics ciblés afin de tenir compte de leur capacité à modifier leurs canaux de recherche d'emploi, en couplant contrôle des refus d'offres d'emploi et contrôle de l'intensité des recherches d'emploi, et enfin en choisissant le bon timing.

\section{Ciblage et effets d'éviction des contrôles}

Aux effets globaux sur l'emploi, qui ne sont pas de même ampleur selon le public concerné, s'ajoutent des effets d'éviction.

Les résultats empiriques divergent quant aux publics pour lesquels les contrôles et les sanctions paraissent les plus efficaces. Les premières études réalisées ont montré que, lorsque le contrôle est ciblé sur des individus dont les perspectives d'emploi sont mauvaises, le contrôle peut être efficace ${ }^{(21)}$. Comme nous l'avons vu, VAN DER BERG et VAN DER KlaAuw (2006) suggèrent que la substitution des canaux de recherche d'emploi (formel/informel) est plus importante pour les personnes les plus quali-

(20) Voir l'article de G. Parent et O. Sautory de ce même numéro pour une présentation des différents effets ex ante et ex post de l'accompagnement.

(21) Voir les revues de littérature antérieures sur les contrôles et les sanctions : FougĖre (2000); CAhuc, Kramarz (2004); FERRACCI (2007). fiées et recommandent donc également de cibler les contrôles sur les personnes ayant de plus faibles perspectives de sortie du chômage. Cependant, cette évaluation porte sur de petits échantillons et les effets ne sont pas toujours significatifs.

Cockx et Dejemeppe (2007) trouvent un effet hétérogène du dispositif $\mathrm{ACR}$, et à l'opposé de celui de VAN DER Berg et VAN DER KlaAuw : en Belgique, la menace de contrôle a un effet important surtout pour les travailleurs les plus employables (22) et l'augmentation de leur probabilité d'emploi est d'autant plus forte que l'on se rapproche de la date prévue du contrôle. Dans la continuité de cette étude, CockX et al. (2011b) montrent que les contrôles sont plus efficaces sur un marché où les offres d'emploi sont abondantes. Dans ce cas, la perspective d'un contrôle peut également avoir un impact positif sur le taux d'embauche des non-qualifiés. Rosholm et al. (2011) identifient pour leur part des effets des contrôles plus importants pour les femmes, tandis que les politiques mixtes (aide à la recherche d'emploi, contrôle, formation ou autre politique active du marché du travail) sont davantage susceptibles d'avoir un impact sur les hommes. Les effets de tous ces programmes sont également légèrement plus importants pour les jeunes (moins de 40 ans) que pour les chômeurs plus âgés (40 ans et plus). Ceci est cohérent avec les résultats de Rosholm et al. (2011), qui observent que les femmes réagissent davantage aux contrôles tandis que les hommes répondent davantage aux effets de menace de l'obligation de participer à des politiques actives du marché du travail.

La littérature n'a pas mis en lumière d'effets de substitution entre demandeurs d'emploi; en revanche, les contrôles conduisent à de forts effets d'éviction vers l'assurance-maladie, la formation et diverses formes d'inactivité. COCKX et Dejemerpe $(2007,2010)$ tentent d'identifier d'éventuels effets de substitution du programme ACR en Belgique : les effets positifs des contrôles sur la population bénéficiaire peuvent entraîner des effets négatifs sur les autres demandeurs d'emploi présents sur le marché du travail, en réduisant la quantité d'offres d'emploi disponibles. En posant un certain nombre d'hypothèses, ils évaluent par différence de différence l'effet du dispositif ACR sur une population non bénéficiaire plus âgée que les groupes ayant suivi le dispositif et le groupe «témoin » (ou de contrôle); ils ne trouvent pas d'impact de la réforme sur cette population «externe». Ils concluent avec prudence que les effets de substitution semblent globalement négligeables.

(22) La menace d'un contrôle augmente la probabilité d'emploi des travailleurs les plus employables de $27 \%$ à $39 \%$. Les auteurs ne trouvent pas d'impact, en revanche, pour les personnes faiblement employables. 
Ils analysent également les éventuels effets d'éviction consécutifs au programme ACR: les demandeurs d'emploi sortiraient de l'assurancechômage non pas pour aller vers l'emploi mais se retrouveraient hors du marché du travail (formation, maladie, etc.). Un effet d'éviction vers l'assurancemaladie est mis en évidence pour les femmes en Wallonie. Cockx et al. (2011a) montrent en outre que l'ACR a stimulé la participation aux formations $(+4$ points de pourcentage en Flandre, +8 points de pourcentage en Wallonie et +11 points de pourcentage à Bruxelles). Pour avoir une vision plus complète des effets d'éviction, les auteurs soulignent la nécessité de tenir compte non seulement de l'indemnisation-chômage, mais également de la fiscalité et des prestations et aides sociales diverses.

Dans certains pays ${ }^{(23)}$, les pensions d'invalidité ont ainsi joué en pratique le rôle d'allocationchômage et l'on a observé des effets d'éviction importants du chômage vers l'invalidité. Ces allocations présentent en effet l'avantage de ne pas être soumises à des conditions de recherche active d'emploi, peuvent être de montant plus élevé que les indemnités-chômage et/ou être perçues durant des périodes plus longues. Les effets d'éviction peuvent donc être relativement importants et nécessitent d'être clairement identifiés sous peine de rendre inefficace toute politique de contrôle.

\section{Les sanctions}

Suite à la constatation d'une infraction aux exigences du service public de l'emploi (SPE), les sanctions appliquées consistent en une réduction, parfois une suppression, des allocations-chômage. Les exigences en matière de recherche d'emploi sont souvent formalisées dans un plan d'actions personnalisé, signé conjointement par le demandeur d'emploi et son conseiller au début de l'épisode de chômage, qui enregistre leurs engagements réciproques. Les sanctions peuvent être courtes (de deux à trois jours), longues (de plusieurs semaines à plusieurs mois) voire, dans certains pays, conduire à la radiation définitive du demandeur d'emploi de l'agence pour l'emploi et du système d'indemnisation (24). Les infractions constatées et sanctionnées peuvent être de natures différentes : défaut d'actualisation de la situation administrative du chômeur ou de son curriculum vitae (CV), conduisant souvent à une sanction courte; refus de participer à

(23) Par exemple les Pays-Bas, qui comptent le plus grand nombre d'inactifs classés comme «invalides» (relativement à la population active), l'Italie et le Royaume-Uni.

(24) Cf. VenN (2012) pour un panorama des cadres réglementaires des pays de l'OCDE et/ou de l'Union européenne. PARENT et al. (2013) situent la France par rapport aux autres pays étudiés au regard de ces différents critères. un programme actif du marché du travail (y compris absence à un entretien avec son conseiller) ou refus d'une offre raisonnable d'emploi, débouchant plutôt sur une sanction longue ${ }^{(25)}$.

\section{Les effets théoriques attendus des sanctions}

VAN DER Berg et al. (2004), LALIVE et al. (2005) et Boone et van Ours (2006) montrent que les sanctions peuvent avoir des effets temporellement distincts. L'effet ex ante ou effet de menace intervient avant même que le manquement aux obligations de recherche ne soit observé, et donc avant qu'une sanction ne soit réellement appliquée au chômeur. On constate que celui-ci augmente ses efforts de recherche d'emploi et réduit son salaire de réserve, augmentant ainsi sa probabilité de reprendre un emploi. Dans la mesure où cette menace peut être associée au fait de "vivre dans un système où l'on peut être sanctionné(26)», elle s'applique à tous les chômeurs, même ceux qui ne sont pas sanctionnés, ni même contrôlés.

Viennent ensuite les effets ex post, qui peuvent prendre plusieurs formes: l'effet d'avertissement intervient dès que le chômeur est notifié par le SPE qu'il est suspecté de manquement à ses obligations et mis sous surveillance accrue; l'effet direct de la sanction survient lorsque la sanction est réellement mise en œuvre, et conduit à une baisse instantanée du salaire de réserve en raison de la baisse voire de la suppression (temporaire ou définitive) de l'allocation-chômage; enfin, les personnes effectivement sanctionnées sont ensuite généralement étroitement surveillées. Elles sont ainsi incitées à se conformer aux exigences de recherche d'emploi pour prévenir de possibles sanctions additionnelles et augmentent l'intensité de leur effort de recherche d'emploi. Il s'agit d'un effet post-sanction, souvent renforcé par la gradation des sanctions et leur plus grande sévérité en cas de récidive dans la plupart des pays (Venn, 2012).

Quelle est l'ampleur respective des différents effets, ex ante comme ex post ? Jan BOONE et Jan C. VAN OURs (2006) estiment pour les Pays-Bas qu'elle dépend de l'intensité des contrôles de la recherche d'emploi. Boone et al. (2009) montrent qu'elle est liée à la nature des sanctions (légères ou sévères, fréquentes ou non, etc.) et de l'offre de travail type. Dans la plupart des cas, la possibilité

(25) Toutes les infractions ne sont pas automatiquement sanctionnées. Si les raisons pour lesquelles on considère que les exigences du SPE ne sont pas remplies sont recevables et bien documentées, dans la plupart des pays, les sanctions sont loin d'être systématiquement appliquées.

(26) Il est difficile de dissocier les effets des contrôles des effets ex ante des sanctions. Notons cependant que l'effet de menace des sanctions est indépendant du fait d'être contrôlé ou non et s'applique à l'ensemble des chômeurs, qu'ils soient contrôlés ou sanctionnés. 
d'être sanctionné (effet ex ante) exerce un rôle plus important que l'application effective de la sanction (effet ex post). Les sanctions stimulent donc les sorties du chômage, surtout au travers de l'effet de menace, comme le montrent la majorité des études sur données empiriques.

\section{Résultats des évaluations empiriques des sanctions}

Si les études menées sur l'effet des sanctions sont nombreuses dans certains pays (États-Unis par exemple), on dispose dans le cas européen de relativement peu d'évaluations, concentrées sur quelques pays (Pays-Bas, Suisse, Danemark et Allemagne). Les analyses qui y ont été réalisées sont révélatrices des difficultés multiples qui émergent lors de l'évaluation de l'impact des sanctions. D'une part, il est nécessaire de distinguer l'effet de la mise en œuvre d'une sanction de l'effet de «vivre dans un système avec sanction » (effet de menace). D'autre part, l'application des sanctions est un processus endogène car la sanction répond à un certain comportement du chômeur. Méthodologiquement, il n'est donc pas possible ni de comparer simplement les taux d'emploi des chômeurs sanctionnés et des chômeurs non sanctionnés, car ces deux groupes diffèrent par leurs caractéristiques et leur comportement, ni de s'appuyer sur des données expérimentales (27). Par ailleurs, les différentes études présentées évaluent des dispositifs de sanctions très hétérogènes, dont l'intensité varie fortement d'un pays à l'autre. En raison de ces forts écarts institutionnels et d'intensité des politiques nationales de sanction, les résultats sont difficilement comparables entre pays.

\section{Les effets de la mise en place d'un système de sanction}

Les premières études se sont attachées à mesurer l'effet de la mise en place de sanctions. Elles font état d'effets positifs importants sur le retour à l'emploi, mais ne distinguent pas l'effet de menace de l'effet de l'application réelle. VAN DER BERG et al. (2004) estiment l'effet des sanctions sur le taux de retour à l'emploi de bénéficiaires de minima sociaux entre 1994 et 1996 à Rotterdam. Les sanctions durent entre un et deux mois et la réduction effective de l'allocation est au maximum de $20 \%$. Les raisons justifiant l'application d'une sanction peuvent être l'insuffisance des activités de recherche d'emploi, une fraude, une démission de l'emploi précédent, un refus de participation à des programmes de formation. Les auteurs mettent en évidence que les sanctions intervenues dans les six premiers mois de l'épisode de chômage augmentent substantiellement les transitions individuelles

(27) Le caractère endogène de l'application des sanctions nécessite ainsi de modéliser simultanément le processus d'application d'une sanction et le processus de retour à l'emploi des demandeurs d'emploi. du système d'assistance sociale vers l'emploi au cours des deux années qui suivent (augmentation de l'ordre de $140 \%$ ). Les taux de transition sont ainsi plus de deux fois supérieurs après mise en œuvre des sanctions que ce qu'ils étaient juste avant. Ils notent également qu'une faible sanction (et la menace d'une punition plus sévère en cas de récidive) peut avoir un impact important sur les comportements de recherche d'emploi. Ils concluent qu'un contrôle plus strict et des conseils de la part des agences sociales accompagnant la mise en œuvre de sanctions pourraient avoir un effet positif sur le comportement de recherche d'emploi de ces bénéficiaires de minima sociaux.

AbBring et al. (2005) estiment, quant à eux, l'effet de sanctions (28) aux Pays-Bas sur le taux de retour à l'emploi de chômeurs entrés au chômage en 1992, indemnisés par l'assurance-chômage, et issus de deux secteurs d'activité particuliers (industries métallurgiques et électroniques; banque et assurance). Les auteurs concluent que suite à l'application d'une sanction, les taux d'emploi augmentent d'environ 35 à $100 \%$ selon les secteurs et le sexe (29). Ils confirment que les effets résultent de deux mécanismes : la baisse des allocations-chômage perçues à cause de la sanction diminue le salaire de réserve des chômeurs et les pousse à accepter des emplois moins bien rémunérés; et l'augmentation de l'intensité de l'effort de recherche augmente la probabilité d'avoir un entretien d'embauche, et donc de retrouver un emploi. Nisar AhMAD et Michael Svarer (2014) analysent, quant à eux, simultanément les effets des sanctions et de plusieurs programmes actifs du marché du travail sur le taux de sortie du chômage des personnes bénéficiaires de l'allocation-chômage entre 2003 et 2005 aux Pays-Bas. Ils montrent que le fait de ne pas prendre en compte la sélectivité des sanctions conduit à sous-estimer leur effet. Selon ces auteurs, le taux de sortie du chômage des bénéficiaires de l'allocation-chômage augmente ainsi de $71 \%$ après l'application d'une sanction. Cependant, des effets d'éviction vers diverses formes d'inactivité sont possibles.

La littérature met aussi en avant un impact négatif des sanctions sur différentes dimensions de la qualité des emplois retrouvés, notamment sur le parcours professionnel ultérieur des chômeurs sanctionnés, le salaire et le temps de travail.

VAN DEN BERG et VIKSTRÖM (2014) mobilisent des données suédoises d'une grande richesse, contenant des informations sur les épisodes de chômage

(28) L'éventail des sanctions va d'une baisse de $5 \%$ de l'indemnité-chômage sur quatre semaines à une diminution de $30 \%$ sur treize semaines.

(29) L'ampleur de ces effets est donc loin d'être négligeable. Cependant, les auteurs reconnaissent que le taux d'emploi des personnes sanctionnées est plutôt faible, et donc que les sanctions conduisent simplement à des taux d'emploi de niveau modéré, sans indication plus précise. 
et les sanctions, sur les salaires horaires, le nombre d'heures travaillées, la qualification de l'emploi retrouvé ainsi que sur les caractéristiques des emplois occupés plusieurs années après l'épisode de chômage. L'étude porte sur la Suède entre 1999 et 2004, et prend en compte des sanctions pour des motifs variés ${ }^{(30)}$. Les sanctions diminuent le salaire horaire obtenu un an après la sortie du chômage (de 3,8\%), augmentent la probabilité d'accepter un emploi à temps partiel (de dix points de pourcentage, soit une augmentation de $15 \%$ ) ou d'une catégorie professionnelle plus basse que celle de l'emploi précédent (conduisant à une perte permanente de capital humain équivalente à quelques semaines d'éducation formelle en moyenne). ARNI et al. (2013) identifient sur données suisses un effet également négatif des sanctions sur la stabilité dans l'emploi et les revenus : les individus sanctionnés subissent une perte nette de revenus équivalente à trente jours sur les deux années suivant le retour à l'emploi (effet ex post). Ils notent par ailleurs que le contrôle plus strict des recherches d'emploi induit une perte supplémentaire de quatre jours de revenus (effet ex ante des sanctions). VAN DEN BERG et al. (2012) évaluent l'effet des sanctions en Allemagne pendant la période 1999-2003, en tenant compte de la probabilité que les chômeurs se déclarent malades ${ }^{(31)}$. Les personnes sanctionnées ont une probabilité plus forte d'entrer de nouveau au chômage, ce qui montre bien que leurs situations d'emploi sont moins stables que celles des personnes n'ayant pas subi de sanction. L'effet sur les salaires est en outre important (baisse de $11 \%$ ) : les emplois occupés par les personnes sanctionnées sont bel et bien plus précaires et moins stables que ceux des personnes non sanctionnées.

Julia SchneIDer (2008) étudie l'effet d'une réforme facilitant et intensifiant l'utilisation des sanctions en Allemagne en 2005. L'auteure n'identifie pas d'effet significatif sur le salaire de réserve (32) des bénéficiaires sanctionnés. Elle en déduit que si les sanctions augmentent les taux de sortie du chômage, c'est principalement parce qu'elles stimulent les efforts de recherche d'emploi. Cependant, l'échantillon des personnes sanctionnées avait un salaire de réserve bas par rapport à l'échelle des salaires allemands, ce qui en rend diffi-

(30) Non-respect des obligations en matière de recherche d'emploi, de participation à des formations ou à des PAMT, refus d'offre raisonnable d'emploi.

(31) Les effets d'éviction sont détaillés plus bas.

(32) L'étude repose sur des données d'enquête; les personnes sélectionnées (sanctionnées comme non sanctionnées) sont interrogées sur l'intégralité de leur parcours professionnel depuis leur sortie du système scolaire (profession, salaire et volume horaire des différents emplois précédents), ainsi que sur le salaire qu'elles souhaiteraient au minimum pour reprendre un emploi. Parce que le salaire de réserve est un concept difficile à appréhender et à évaluer par les personnes elles-mêmes, ce salaire de réserve autodéclaré est dans un second temps ajusté pour tenir compte des éventuels biais de déclaration. cile une éventuelle baisse. Si ces personnes restent au chômage, c'est plutôt, selon l'auteure, en raison d'une pénurie d'offres d'emploi en direction de ce public qu'en raison de leur salaire de réserve.

\section{Les effets distincts de la menace et de l'application des sanctions}

Au-delà de ces effets globaux sur le retour à l'emploi, les articles plus récents tentent d'entrer dans la «boîte noire» des sanctions pour analyser dans le détail la temporalité des mécanismes à l'œuvre lors de leur application et identifier à quel moment du parcours du demandeur d'emploi une sanction produit les effets les plus importants.

Le poids relatif des effets ex ante (effet de menace de sanction) et des effets ex post (effet de l'application effective d'une sanction) est variable, et dépend de la structure du système de sanction comme de la vitalité du marché du travail local. La nouveauté des articles de LALIVE et al. (2005) et ARNi et al. (2013) vient du fait que les auteurs disposent de données suisses contenant, outre la date d'application d'une sanction, des informations précises sur la date à laquelle le demandeur d'emploi a reçu notification de la possibilité d'être sanctionné prochainement: le chômeur est ainsi informé dans un premier temps qu'il est suspecté de violation des critères de recherche et qu'une sanction pourra être appliquée si un effort de recherche insuffisant est constaté par son conseiller. Ces données permettent aux auteurs d'identifier, sur la période 1997-1999, des effets positifs (33) à la fois de la menace de sanction (ex ante), qui augmente le taux de retour à l'emploi de $25 \%$ environ, et de l'application effective d'une sanction (ex post), qui le fait progresser de $20 \%$ supplémentaires. La mise en œuvre de sanctions diminue ainsi la durée moyenne de chômage d'environ trois semaines (LALIVE et al., 2005). Utilisant une méthode légèrement différente, ARNI et al. (2013) aboutissent à des résultats du même ordre de grandeur, puisque l'effet de menace augmente de $17 \%$ les sorties vers l'emploi tandis que l'application effective de la sanction y ajoute $16 \%$ supplémentaires. Les auteurs indiquent que leurs résultats diffèrent des premières études d'ABBring et al. (2005) et VAN DER BERG et al. (2004), qui ne disposaient pas de l'information sur l'avertissement de la sanction et ne pouvaient donc distinguer effet de menace et effet de la sanction.

Cockx et al. (2011a) montrent dans le cadre du dispositif belge ACR ( $c f$. supra) que la menace de sanctions augmente sensiblement les taux de reprise d'emploi des demandeurs d'emploi (de l'ordre de 16 points de pourcentage en Flandre) avant même les entretiens individuels de contrôle avec les conseillers et l'application éventuelle d'une sanction. La

(33) Les auteurs n'identifient pas d'effets hétérogènes des sanctions selon le sexe ou l'âge des populations. 
simple lettre d'avertissement conduit à une intensification des efforts de recherche d'emploi et des transitions vers l'emploi. Cet effet de menace représenterait selon eux $92 \%$ de l'effet total des sanctions.

Barbara Hofmann (2010) évalue l'effet d'une modification de politique publique en Allemagne en 2003, qui s'est traduite par un renforcement des sanctions. L'application très inégale de ce changement de politique dans les 180 districts allemands (certains ayant augmenté très significativement la fréquence des sanctions et d'autres non) lui permet de mettre en place une stratégie d'évaluation d'impact basée sur cette expérience naturelle pour estimer l'effet ex ante des sanctions. L'étude conclut à l'existence d'un fort effet de la menace de sanction sur le taux de transition vers l'emploi, qui est compris entre six et douze points de pourcentage un an après l'entrée au chômage. Dans un article plus récent, l'auteure analyse les effets ex post de l'application de sanctions fortes en Allemagne entre 2000 et 2001 (suppression totale de l'allocation-chômage pendant douze semaines en cas de refus d'une formation ou d'un PAMT), par méthode d'appariement ${ }^{(34)}$. Le groupe de personnes sanctionnées a des taux d'emploi non subventionné(35) supérieurs de cinq à dix points de pourcentage (douze mois après la sanction) par rapport au groupe de contrôle, qui n'a pas subi de sanction (HoFMANN, 2012).

AbBring et al. (2005) comme VAN DER KLAAUw et van Ours (2013) pour les Pays-Bas, et Müller et SteIner (2008) pour l'Allemagne, mettent en évidence des effets plus forts des sanctions pour les femmes que pour les hommes. Au contraire, Ahmad et Svarer (2014) identifient des effets plus élevés pour les hommes que pour les femmes aux Pays-Bas. Ces résultats contradictoires d'une étude à l'autre sont surtout le signe que l'impact des sanctions dépend fortement du contexte institutionnel, des possibilités d'éviction par l'entrée en formation ou par les sorties du marché du travail(36), mais également de la nature et de l'intensité des sanctions évaluées.

\section{La sévérité et le timing des sanctions}

Des sanctions plus sévères ou appliquées plus systématiquement ont un impact accru sur le retour à l'emploi. Le renforcement des critères d'éligibilité à l'assurance-chômage combiné à un

(34) Cette méthode associe à des personnes sanctionnées dans les zones ayant appliqué strictement le renforcement des sanctions, des personnes non sanctionnées dans les zones n'ayant pas ou peu intensifié les sanctions, mais qui l'auraient été si elles avaient résidé dans une zone stricte.

(35) Emploi n'ayant pas bénéficié d'une aide publique à l'embauche.

(36) Comme pour les contrôles, les effets d'éviction de l'assurance-chômage vers l'assurance-maladie ou les systèmes d'invalidité sont importants dans certains pays européens (Europe du Nord principalement). recours plus fréquent aux sanctions et une augmentation du nombre de bénéficiaires des PAMT ont contribué à la baisse du chômage et au succès du modèle danois de «flexisécurité» entre le milieu des années 1990 et la crise de 2008 (Rosholm et al., 2011). Svarer (2011) mobilise une base administrative très riche couvrant la période 2003-2005 au Danemark. Il estime simultanément le processus de sortie du chômage et le processus de sanction, autorisant les deux à être interdépendants. Il montre que les sanctions augmentent la probabilité de retrouver un emploi de plus de $80 \%$ dans le mois suivant lorsque l'on tient compte de l'endogénéité des sanctions (30\% seulement dans le cas de l'estimation naïve) et que cet effet direct progresse avec la sévérité de la sanction : en effet, les sanctions longues sont plus efficaces que les sanctions courtes. Les sanctions courtes considérées ici sont des sanctions de deux à trois jours uniquement (pénalisant de petites infractions aux exigences du service public de l'emploi comme la non-actualisation de sa situation administrative ou de son CV par exemple) par opposition à des sanctions longues de trois semaines (en cas de refus d'une offre raisonnable d'emploi ou de refus de participation à un programme actif du marché du travail) ${ }^{(37)}$. Il ne s'agit donc pas de différence de sévérité d'une sanction prononcée pour un même manquement.

LALIVE et al. (2005) constatent (sans le démontrer de manière causale) que plus la sanction est stricte, plus la durée des épisodes de chômage des personnes non sanctionnées est courte, laissant à penser que les effets de menace sont d'autant plus importants en Suisse que la sanction encourue est sévère. BOOCKMANN et al. (2009) utilisent, quant à eux, la variation de l'intensité d'application des sanctions dans les 439 agences de l'emploi allemandes (38) (entre 2006 et 2007) pour estimer l'effet d'une utilisation accrue des sanctions. Ils estiment ainsi l'effet qu'aurait une sanction sur des personnes qui n'ont pas été sanctionnées dans les agences les moins sévères (malgré un manquement à leurs obligations), mais qui l'auraient été dans des agences plus strictes. Ici, l'effet moyen sur l'ensemble de la population peut être interprété comme l'impact

(37) À noter que les sanctions sévères dont il est question dans cette étude restent très inférieures aux sanctions classiques prévues en France en cas d'absence à une convocation ou à un rendez-vous médical, ou en cas de refus de deux offres raisonnables d'emploi ou de refus d'élaboration ou d'actualisation du projet personnalisé d'accès à l'emploi (PPAE, qui prévoit la suppression de l'allocation pendant deux mois); les sanctions danoises sont plus proches des sanctions considérées comme légères en France (quinze jours en cas d'insuffisance de recherche d'emploi, de refus d'une action de formation, d'un contrat d'apprentissage ou de professionnalisation, d'une action de réinsertion ou d'un contrat aidé).

(38) Les auteurs retiennent l'hypothèse d'exogénéité de ces différences d'intensité dans l'application des sanctions entre agences. La validité de l'instrument semble vérifiée : en effet, les agences du groupe de traitement et de contrôle ne se situent pas dans des bassins locaux d'emploi différents, en moyenne. 
qu'aurait une généralisation de l'utilisation des sanctions (si les agences indulgentes se comportaient de la même manière que les agences plus sévères). Pour les personnes sanctionnées dans les agences strictes et non sanctionnées dans les agences souples, l'application d'une sanction augmenterait très fortement leur probabilité d'accepter un emploi (dans les cinq mois qui suivent la perte de l'allocation) : cette probabilité passe, pour la situation de référence, de $20 \%$ à $70 \%$. Les auteurs concluent qu'une utilisation plus intensive des sanctions par les agences pour l'emploi contribuerait à rendre l'activation du marché du travail plus efficace en Allemagne et accroîtrait substantiellement les transitions vers l'emploi.

Des sanctions même réduites peuvent avoir un effet important sur les comportements des chômeurs, en raison de la persistance de leur effet (effets d'hystérèse). Ainsi, les résultats négatifs observés par VAN DER BERG et VIKSTRÖM (2014) sur les salaires (- 3,8\%, cf. supra) et la probabilité d'accepter un emploi à temps partiel $(+10,3 \%)$ un an après l'application des sanctions se confirment lorsque les auteurs réitèrent leur analyse à un horizon plus lointain: deux et trois ans après la sanction, les effets négatifs sur les salaires retrouvés persistent et augmentent même légèrement (- $4,7 \%$ d'écart de salaire observé trois ans après la sanction). L'impact des sanctions se prolonge donc bien au-delà de la période de sanction elle-même et peut conduire à des trajectoires professionnelles plus erratiques et à des emplois moins bien rémunérés à long terme.

Kai-Uwe MüLler (2007) constate qu'en Allemagne les sanctions surviennent davantage dans les six premiers mois de l'épisode de chômage. Cette temporalité est-elle la plus efficace? MÜLLER et STEINER (2008) approfondissent cette question à partir de données contenant des informations précises sur le timing et les durées à la fois des épisodes de chômage et des sanctions. Ils se concentrent sur les sanctions pour refus d'offre raisonnable d'emploi ou de participation à un PAMT. Ces sanctions ont un fort effet positif, immédiat ou à court terme, sur la probabilité de retour à l'emploi. L'effet d'une sanction est plus élevé lorsqu'elle est appliquée tôt dans l'épisode de chômage : par exemple, pour les hommes en Allemagne de l'Ouest, la différence de probabilité de sortie du chômage entre le groupe de chômeurs sanctionnés et le groupe de contrôle est de huit points de pourcentage si la sanction est imposée dans les trois premiers mois. Une sanction administrée quinze mois après le début de l'épisode de chômage ne réduit en revanche pas sa durée.

Cela étant, la prise en compte d'aspects plus qualitatifs et psychologiques nuance le consensus global en faveur des sanctions, comme le montre une enquête qualitative menée au Royaume-Uni sur l'impact de sanctions très lourdes (suspension d'allocation pendant vingt-six semaines) pour refus de participation à un PAMT. Si un certain nombre de demandeurs d'emploi sanctionnés ont ensuite intensifié leurs efforts de recherche d'emploi et ont repris rapidement un travail de courte durée ou à temps partiel, ceux qui sont confrontés à de véritables barrières au retour à l'emploi (transport, garde d'enfant, aptitude à décrocher un emploi, difficultés d'apprentissage, illettrisme, casier judiciaire, problème de drogue ou d'alcool), n'ont pas pour autant accéléré leurs recherches. Pour ces chômeurs en effet, les sanctions sont stressantes, déprimantes et, associées à un sentiment de honte, peuvent entraîner une perte de confiance en soi dommageable, notamment, pour de futures recherches sur le marché du travail. Certains conseillers du SPE pensent ainsi qu'il serait utile d'étendre la période "gateway» (période d'aide à la recherche d'emploi avant l'obligation de participer à un PAMT) pour des demandeurs d'emploi ayant besoin de plus de temps pour retrouver un emploi. Les sanctions ne constitueraient peut-être pas une réponse adaptée pour ces populations vulnérables car elles peuvent avoir des effets néfastes à long terme.

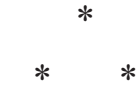

Les évaluations empiriques présentées dans la revue de littérature que nous venons de proposer convergent dans leurs principaux résultats. Premièrement, les contrôles comme les sanctions ont des effets positifs sur le retour à l'emploi, mais négatifs sur la qualité des emplois retrouvés. Ces mécanismes d'activation inciteraient donc les demandeurs d'emploi à accepter des emplois moins stables et moins bien rémunérés. Ils peuvent également avoir des effets pénalisants sur le parcours professionnel ultérieur des personnes sanctionnées. Deuxièmement, les contrôles comme les sanctions sont assortis d'effets de menace importants : ainsi la simple menace de contrôle/sanction modifie les comportements de tous les demandeurs d'emploi (sanctionnés comme non sanctionnés) et 1'application réelle d'une sanction n'est pas, en elle-même, l'élément le plus efficace du système d'activation. Les poids relatifs des effets ex ante (effet de menace d'une sanction) et des effets ex post (effet de l'application effective d'une sanction) sont cependant variables et dépendent de la durée, de l'ampleur et du timing des sanctions. Troisièmement, l'analyse de la mise en place de ces politiques montre que les contrôles modifient les comportements de recherche d'emploi, avec notamment une substitution des canaux informels par les canaux formels, ainsi que le timing de la recherche d'emploi et son intensité.

Les résultats empiriques divergent quant aux publics pour lesquels les contrôles ou les sanctions paraissent les plus efficaces. Les effets positifs constatés sur les bénéficiaires ne semblent pas être 
observés au détriment des non-bénéficiaires (peu ou pas d'effet de substitution). En revanche, les contrôles comme les sanctions s'accompagnent d'effets d'éviction du chômage vers l'assurancemaladie, la formation et diverses formes d'inactivité.

Par ailleurs, l'effet des sanctions perdure au-delà de la période de réduction/suppression des allocations (effets d'hystérèse). L'efficacité des contrôles et des sanctions dépend tant de la demande que de l'offre de travail : en effet, le dynamisme du marché du travail local (nombre d'offres d'emploi disponibles sur ce marché) et le niveau structurel du taux de chômage ont un effet déterminant sur l'impact que peuvent avoir sanctions comme contrôles de la recherche d'emploi sur les taux de retour à l'emploi des chômeurs.

Néanmoins, il convient d'être prudent, d'une part, quant à l'interprétation des résultats obtenus sur l'effet des sanctions dans d'autres pays européens et, d'autre part, quant à leur éventuelle transposition dans le cas français, car les sanctions ne sont pas d'intensité comparable en France et dans le reste de l'Europe ${ }^{(39)}$. Ainsi, le rapport AlduY (2011, p. 117) de la mission d'information du Sénat sur Pôle emploi, illustre une spécificité française :

«Les manquements qu'on serait spontanément tenté de considérer comme les plus graves aux devoirs des demandeurs d'emploi (insuffisance des actes de recherche d'emploi, refus d'une proposition concrète d'emploi ou de formation présentée par le service public de l'emploi) débouchent, le plus souvent, sur la sanction la plus douce dans l'échelle des peines : dans $90 \%$ des cas, ils se traduisent par une radiation de quinze jours. À l'inverse, la non-réponse à une convocation, qui justifie plus de $90 \%$ des radiations, aboutit à une radiation de deux mois.»

Des recherches existantes en la matière émergent un certain nombre de recommandations de politique publique. Contrôler l'effort de recherche d'emploi des chômeurs est plus efficace que contrôler leurs refus d'offres d'emploi. Cependant dans le cas français, il s'agirait non seulement de contrôler davantage l'effort de recherche d'emploi, mais également de faire en sorte qu'un refus de proposition d'emploi - concrète et en adéquation avec le métier recherché - conduise à une sanction plus lourde qu'actuellement, et de moins pénaliser, en contrepartie, les non-réponses à une convocation ou à un entretien. Ensuite, il importe de revoir le timing des contrôles et des sanctions, et de tenir compte de l'hétérogénéité des réactions des différentes populations à ces incitations, comme par exemple leur plus ou moins grande facilité à substituer des canaux de recherche d'emploi, ainsi que l'efficacité relative des canaux formels ou informels pour les populations ciblées. Enfin, il semble préférable d'associer contrôles/sanctions à une aide efficace à la recherche d'emploi, car la complémentarité entre ces deux mécanismes d'activation ( «carrot and stick» pour reprendre l'expression de VAN DER KLAAUW et VAN Ours, 2013) conduit à de meilleurs taux d'emploi que l'un ou l'autre pris séparément ${ }^{(40)}$.

\section{Bibliographie}

Abbring J., van den Berg G., van Ours J. (2005), «The effect of unemployment insurance sanctions on the transition rate from unemployment to employment», The economic journal, vol. 115, $\mathrm{n}^{\circ} 505$, pp. 602-630.

Acemoglu D. (2001), «Good jobs versus bad jobs», Journal of labor economics, vol. 19, $\mathrm{n}^{\circ}$ 1, pp. 1-21.

Ahmad N., Svarer M. (2014), The effect of sanctions and active labour market programmes on the exit rate from unemployment, mimeo August, University of Aarhus.

AlduY (2011), Rapport d'information de la mission commune d'information relative à Pôle emploi, $\mathrm{n}^{\circ} 713$, Paris, Sénat.

Arni P., Lalive R., van Ours J. (2013), «How effective are unemployment benefit sanctions? Looking beyond unemployment exit», Journal of applied econometrics, vol. $28, \mathrm{n}^{\circ} 7$, pp. 1153-1178.

(39) Rappelons que les sanctions considérées comme légères dans certains pays sont de l'ordre de deux à trois jours, contre une quinzaine en France, tandis que les sanctions sévères peuvent consister en deux ou trois semaines de suppression d'allocation quand elles atteignent deux mois en France.
Ashenfelter O., Ashmore D., Deschênes O. (2005), «Do unemployment insurance recipients actively seek work? Evidence from randomized trials in four U.S. states », Journal of econometrics, vol. 125, n 1-2, pp. 53-75.

Boockmann B., Thomsen S., Walter T. (2009), «Intensifying the use of benefit sanctions: an effective tool to shorten welfare receipt and speed up transitions to employment?», IZA Discussion paper, $\mathrm{n}^{\circ} 4580$.

Boone J., Sadrieh A., van Ours J. (2009), «Experiments on unemployment benefit sanctions and job search behavior», European economic review, vol. 53, $\mathrm{n}^{\circ} 8$, pp. 937-951.

BOONE J., van Ours J. (2006), «Modelling financial incentives to get unemployed back to work», Journal of institutional and theoretical economics, vol. 162, $\mathrm{n}^{\circ} 2$, pp. 227-252.

(40) Comme le soulignaient déjà les revues de littérature antérieures (FougĖre, 2000; CAHUC et Kramarz, 2004; FerracCI, 2007). 
Cahuc P., Kramarz F. (2004), De la précarité à la mobilité: vers une sécurité sociale professionnelle, Rapport au ministre de l'Économie, des Finances et de l'Industrie et au ministère de l'Emploi, du Travail et de la Cohésion sociale, Paris, La Documentation française.

Cockx B., Dejemeppe M. (2007), «Is the notification of monitoring a threat to the unemployed? A regression discontinuity approach», IZA Discussion paper, $\mathrm{n}^{\circ} 2854$.

Cockx B., Dejemeppe M. (2010), «The threat of monitoring job search. A discontinuity design», IZA Discussion paper, $\mathrm{n}^{\circ} 5337$.

Cockx B., Dejemeppe M., Launov A., van der Linden B. (2011a), «Monitoring, sanctions and front-loading of job search in a non-stationary model», IZA Discussion paper, $\mathrm{n}^{\circ} 6181$

Cockx B., Dejemeppe M., VAn der Linden B. (2011b), Évaluation de l'activation du comportement de recherche d'emploi, Série «Société et Avenir», Gent, Academia Press.

Ferracci M. (2007), «Améliorer le service public de l'emploi: ce que disent les faits», Revue française d'économie, vol. 21, n 3, pp. 75-135.

FougĖre D. (2000), «Accompagnement des chômeurs et sanctions : leurs effets sur le retour à l'emploi», in Pisani-Ferry J. (dir.), Plein emploi, Rapport du Conseil d'analyse économique, $\mathrm{n}^{\circ} 30$, pp. 313-340.

Friedman M. (1962), Capitalism and freedom, Chicago, University of Chicago Press.

Hofmann B. (2010), Tightening the use of unemployment benefit sanctions - does it speed up the exit to work? Evidence from a policy change, mimeo IAB.

Hofmann B. (2012), "Short- and long-term ex-post effects of unemployment insurance sanctions », Journal of economics and statistics, vol. 232, n ${ }^{\circ} 1$, pp. 31-60.

Labrousse A., Zamora P. (coord.) (2013), «Expérimentations de terrain et politiques publiques $\mathrm{du}$ travail et de l'emploi. Apports récents et mises en perspective», Travail et emploi, $\mathrm{n}^{\circ} 135$.

Lalive R., van Ours J., Zweimüller J. (2005), «The effect of benefit sanction on the duration of unemployment», Journal of the European economic association, vol. 3, $\mathrm{n}^{\circ} 6$, pp. 1386-1417.

Magniadas J. (2008), «Le long chemin vers la reconnaissance des chômeurs », Les cahiers de l'institut CGT d'histoire sociale, $\mathrm{n}^{\circ} 105$, pp. 6-11.

MC VICAR D. (2008), «Job search monitoring intensity, unemployment exit and job entry: quasi experimental evidence from the UK», Labour economics, vol. 15, $\mathrm{n}^{\circ}$ 6, pp. 1451-146.

Meyer B. (1995), «Lessons from the US unemployment insurance experiments », Journal of economic literature, vol. $33, \mathrm{n}^{\circ} 1$, pp. 91-131.

Mortensen D. (1986), «Job search and labor market analysis », in Ashenfelter O., Layard R. (ed.), Handbook of labor economics, vol. 2, Amsterdam, North-Holland, pp. 849-919.

MüLlER K. (2007), «Observed and unobserved determinants of unemployment insurance benefit sanctions in Germany. Evidence from matched individual and regional administrative data», WZB Discussion paper, $\mathrm{n}^{\circ}$ 2007-107.

MüLler K., STEINER V. (2008), «Imposed benefit sanctions and the unemployment-to-employment transition. The German experience», DIW Discussion paper, $\mathrm{n}^{\circ} 792$.

OCDE (2000), Perspectives de l'emploi de l'OCDE 2000, Paris, Éditions OCDE.

OCDE (2006), Perspectives de l'emploi de l'OCDE 2006 : stimuler l'emploi et les revenus, Paris, Éditions OCDE.

OCDE (2009), Perspectives de l'emploi de l'OCDE 2009 : faire face à la crise de l'emploi, Paris, Éditions OCDE.

OCDE (2012), Perspectives de l'emploi de l'OCDE 2012, Paris, Éditions OCDE.

Parent G., Sautory O., Desplatz R. (2013), «L'accompagnement des demandeurs d'emploi : enseignements des évaluations», Documents d'études, $\mathrm{n}^{\circ} 178$, Dares.

Petrongolo B. (2009), «The long-term effects of job search requirements: evidence from the UK JSA reform», Journal of public economics, vol. 93, $\mathrm{n}^{\circ}$ 1-2, pp. 1234-1253.

Rosholm J., Svarer M., Vikström M. (2011), «The relative efficiency of active labor market policies: evidence from a social experiment and non-parametric methods», Working paper, $\mathrm{n}^{\circ}$ 2011-7, IFAU.

SCHNEIDER J. (2008), « The effect of unemployment benefit II sanctions on reservation wages », IAB Discussion paper, $\mathrm{n}^{\circ} 19-2008$.

Stigler G. (1962), «The intellectual and the marketplace », Selected papers, $\mathrm{n}^{\circ} 3$, Chicago, University of Chicago graduate school of business.

SVARER M. (2011), «The effect of sanctions on exit from unemployment: evidence from Denmark», Economica, vol. $78, \mathrm{n}^{\circ} 312$, pp. 751-778.

VAN DEN BERG G. (1990), «Search behaviour, transitions to non-participation and the duration of unemployment », The Economic journal, vol. 100, n 402, pp. 842-865.

van den Berg G., Hofmann B., Uhlendor A. (2012), The role of sickness in the evaluation of job search assistance and sanctions on unemployment and the subsequent job quality, mimeo, ESPE Conference.

van den Berg G., van der KlaAuw B. (2006), «Counseling and monitoring of unemployed workers: theory and evidence from a controlled social experiment», International economic review, vol. 47, n 3, pp. 895-936. 
VAN den Berg G., van der KlaAuw B. (2013), «Structural empirical evaluation of job search monitoring», IZA Discussion paper, $\mathrm{n}^{\circ} 7740$.

van den Berg G., van der KlaAuw B., van Ours J. (2004), «Punitive sanctions and the transition rate from welfare to work», Journal of labor economics, vol. 22, $\mathrm{n}^{\circ} 1$, pp. 211-241.

VAN DEN Berg G., Vikström J. (2014), «Monitoring job offer decisions, punishments, exit to work, and job quality", The Scandinavian journal of economics, vol. $116, \mathrm{n}^{\circ} 2$, pp. 284-334. van der Klaauw B., van Ours J. (2013), «Carrot and stick: how reemployment bonuses and benefit sanctions affect exit rates from welfare», Journal of applied econometrics, vol. 28, ${ }^{\circ}$ 2, pp. 275-296.

VENN D. (2012), «Eligibility criteria for unemployment benefits, quantitative indicators for OECD and EU countries», OECD social, employment and migration working papers, $\mathrm{n}^{\circ} 131$.

Wunsch C. (2010), Optimal monitoring and sanctioning systems, mimeo, Swiss institute for empirical economic research, University of St. Gallen. 